\title{
Was können biologische Marker für die Verhaltenstherapie leisten?
}

\author{
Sarah Schumacher ${ }^{\mathrm{a}}$ Susanne Fischer ${ }^{\mathrm{b}}$ \\ ${ }^{a}$ Arbeitsbereich Klinisch-Psychologische Intervention, Fachbereich Erziehungswissenschaft und Psychologie, \\ Freie Universität Berlin, Berlin, Deutschland; 'blinische Psychologie und Psychotherapie, Psychologisches Institut, \\ Universität Zürich, Zürich, Schweiz
}

Die moderne Klinische Psychologie rekurriert ganz selbstverständlich auf multifaktorielle Störungsmodelle, die biologische, psychologische und soziale Determinanten psychischer Störungen berücksichtigen [Engel, 1977]. In der kognitiv-verhaltenstherapeutischen Praxis werden biologische Faktoren hingegen eher am Rande (z.B. im Rahmen der Psychoedukation) berücksichtigt; es sind vor allem die psychologischen Aspekte, wie kognitive Verzerrungen bei depressiven Störungen oder das Vermeidungsverhalten bei Angststörungen, die bei der Diagnostik bedacht und von Psychotherapeut*innen als Ansatzpunkte für mögliche Veränderungen wahrgenommen werden. Diese Fokussierung ergibt sich natürlicherweise aus der psychologisch ausgerichteten Ausbildung von kognitiven Verhaltenstherapeut*innen. Gleichzeitig scheint eine Marginalisierung biologischer Faktoren insofern bemerkenswert, als biologische Prozesse nicht nur in der Entstehung psychischer Störungen eine wichtige Rolle spielen, sondern als sich die Symptome psychischer Störungen immer auch auf der körperlichen Ebene manifestieren. Sollten Psychotherapeut*innen also dem biologischen Teil des biopsychosozialen Modells mehr Aufmerksamkeit schenken?

In der klinisch-psychologischen Forschung ist dies bereits geschehen: Längst haben hier Messmethoden aus Nachbardisziplinen wie der biologischen Psychologie oder den Neurowissenschaften Einzug in die Forschung erhalten. Dies bedeutet, dass sich immer mehr klinische Psycholog*innen in ihrer Arbeit mit der Funktion von hormonellen Systemen, mit neurobiologischen Prozessen oder mit (epi-)genetischen Grundlagen von psychischen Störungen beschäftigen. Während ab den 1980er Jahren vor allem die pathophysiologische Relevanz und das diagnostische Potenzial von biologischen Markern [z.B. Dexamethason-Suppressionstest bei depressiven Störungen; Carroll, 1982] im Zentrum eines wachsenden wissenschaftlichen Interesses stand, welches nach wie vor an Entwicklungen wie den Research Domain Criteria des US National Institute of Mental Health (NIMH) ablesbar ist [Insel et al., 2010], hat in jüngerer Zeit eine Hinwendung zur Erforschung von biologischen Indikatoren und Prädiktoren erfolgreicher Psychotherapien stattgefunden. Dieser Forschungszweig birgt das Potenzial, zukünftig die Wirksamkeit der kognitiven Verhaltenstherapie (KVT) zu verbessern, indem er sich dem "what works for whom and why" widmet. Entsprechend dürften gerade diese neueren Entwicklungen auch für Therapeut*innen und Patient*innen von Interesse sein.

Das Ziel dieses Themenhefts ist es, eine Übersicht über die neuesten Erkenntnisse aus dem rapide wachsenden Forschungsfeld der biologisch orientierten Psychotherapieforschung darzubieten. Hierbei soll auch aufgezeigt werden, inwieweit zugehörige Befunde für Therapeut*innen bereits heute nutzbar gemacht werden können. Die in diesem Heft enthaltenen Artikel können dabei grob in zwei Gruppen unterteilt werden: Während die Arbeiten von Stoffel et al., Euteneuer et al. und Karrasch et al. die biologischen Wirkweisen und Ergebnisse von 
erfolgreichen kognitiv-behavioralen Interventionen beschreiben, sind die Beiträge von Schaumburg et al., Hilbert und Lueken und Engel et al. mit der Vorhersagekraft von zu Beginn der psychotherapeutischen Behandlung erhobenen biologischen Merkmalen befasst.

Stoffel et al. gehen im ersten Beitrag der Frage nach, inwieweit mit Stress assoziierte epigenetische Signaturen durch kognitiv-behaviorale Interventionen rückgängig gemacht werden können. Die Autor*innen ziehen zunächst Befunde aus dem Forschungsfeld der "behavioural epigenetics" [z.B. Lester et al., 2011] heran, die nahelegen, dass Umwelteinflüsse (z.B. Kindheitstraumata, arbeitsbezogener Stress) Veränderungen in Methylierungsmustern von wichtigen, die körperliche Stressreaktion modulierenden, Genen herbeiführen und so - via ebenfalls veränderter Genexpression - einen direkten Einfluss auf die Stimmung und die kognitive Funktionsfähigkeit von Personen nehmen können. Anhand eigener empirischer Untersuchungen an Studierenden und Arbeitnehmer*innen zeigen sie in einem nächsten Schritt auf, dass Stressbewältigungstrainings bereits nach einer Woche die Methylierungsmuster von serotonergen (SLC6A4) und Bestandteile der Hypothalamus-Hypophysen-Nebennierenrinden-Achse enkodierenden (NR3C1, FKBP5) Genen beeinflussen. Diese Studien beschreiben auf eindrückliche Weise die Wirksamkeit und Wirkweisen kognitivbehavioraler Maßnahmen in einem präventiven Kontext.

Euteneuer et al. befassen sich in ihrem Artikel mit einer Reihe von Auffälligkeiten in stress-responsiven körperlichen Systemen bei Patient*innen mit einer Major Depression, die erwiesenermaßen das Risiko für kardiovaskuläre Erkrankungen erhöhen. Aus der darauf basierenden systematischen Übersichtsarbeit der Autor*innen ergeben sich erste Hinweise darauf, dass eine KVT diesbezüglich einen protektiven Einfluss ausüben kann. So deuten die Ergebnisse auf eine Reduktion pro-inflammatorischer Zytokine zum Therapieende hin, was den bei depressiven Personen oft zu beobachtenden subklinisch erhöhten Entzündungswerten und dem damit einhergehenden "sickness behaviour" [z.B. Dantzer et al., 2008] entgegenwirken könnte. Diese Studie verweist auf den hochrelevanten Sachverhalt, dass die KVT nicht nur Symptome von psychischen Störungen zu lindern vermag, sondern auch positiv auf den körperlichen Gesundheitszustand einwirken und so potentiellen Folgeerkrankungen vorbeugen kann.

Karrasch et al. fokussieren auf die biologischen Effekte der KVT bei körperlichen Erkrankungen. Die Autor*innen verweisen zunächst auf Befunde, die nahelegen, dass chronischer und traumatischer Stress Veränderungen im Immunsystem und einen Überschuss an reaktiven Sauerstoffverbindungen generiert, was wiederum in Schäden an der DNA, an Proteinen und an Lipiden [sog. "oxidativer Stress"; z.B. Sies et al., 2017] resultieren und so den Alterungsprozess beschleunigen kann. Im Folgenden fassen die Autor*innen Studien zusammen, die untersuchen, ob ebendiese stressbezogenen Folgen durch Mind-Body-Interventionen verändert werden können. Sie zeigen auf, dass zugehörige Programme, wie Mindfulness-Based Stress Reduction, oder therapeutische Techniken, wie Hypnose, bei Patient*innen mit somatomedizinischen Erkrankungen wie HIV, Krebs oder ulzerativer Colitis förderliche Effekte auf Immunparameter haben können. Dieser Beitrag unterstreicht überzeugend die Wirksamkeit kognitiv-behavioraler Interventionen in einem verhaltensmedizinischen Kontext und liefert Hinweise auf mögliche Wirkmechanismen.

Hilbert und Lueken beleuchten in ihrem Artikel die Rolle der prädiktiven Analytik in der Klinischen Psychologie. Sie erläutern dabei zunächst die Methode des maschinellen Lernens [z.B. Dwyer et al., 2018], wobei im Kontext von Psychotherapie Indikatoren aus verschiedenen Domänen (z.B. klinische Merkmale, funktionelle Bildgebungsdaten) derart verknüpft werden, dass schließlich jede Variablenkombination die Zugehörigkeit zu einer bestimmten Klasse (z.B. Diagnose) vorhersagen kann. Die Autor*innen illustrieren den potentiellen Nutzen solcher Modelle unter anderem am Beispiel einer eigenen Untersuchung, bei der die symptomatisch überlappenden Störungsbilder der Major Depression und der generalisierten Angststörung anhand einer Kombination von neuroanatomischen, endokrinen und Selbstberichtsdaten besser voneinander abgegrenzt werden konnten als anhand monomodaler Daten. Diese hochaktuellen Befunde stellen den inkrementellen Nutzen biologischer Marker im differentialdiagnostischen Prozess heraus.

Schaumburg et al. konzentrieren sich in ihrem Beitrag auf mögliche genetische Prädiktoren von Psychotherapieerfolg. Hierzu werden verschiedene Befunde aus dem vielversprechenden Forschungsfeld der "therapygenetics" [z.B. Eley, 2014] angeführt. Die Autor*innen liefern dabei Hinweise auf die bisher noch bestehenden methodischen Limitationen von Kandidatengenstudien und genomweiten Assoziationsstudien und zeigen Wege für die zukünftige Forschung auf. So kranken erste Versuche, anhand einzelner oder mehrerer Gene den Behandlungserfolg vorherzusagen, vor allem an zu kleinen und/oder heterogenen Stichproben, welche eine Detektion der zu erwartenden kleinen Effekte unmöglich machen. Eine vielversprechende Entwicklung stellen hingegen polygene Scores dar, die der multifaktoriellen Determiniertheit von psychischen Störungen Rechnung tragen. Zusammenfassend demonstrieren die Autor*innen auf eindrucksvolle Weise das (noch längst nicht ausgeschöpfte) indikative Potenzial genetischer Marker.

Engel et al. nehmen die Bedeutung des sozialen Neuropeptids Oxytocin in Bezug auf wichtige Wirkfaktoren der Psychotherapie in Augenschein. Hierzu untersuchten
Verhaltenstherapie 2020;30:5-7 DOI: $10.1159 / 000506426$
Schumacher/Fischer 
die Autor*innen in einer aufwendigen Studie 35 Bundeswehrsoldaten mit einer posttraumatischen Belastungsstörung, die an einer fünfwöchigen internetbasierten KVT teilnahmen. Obwohl statistisch nicht signifikant, zeigte sich bei Patienten mit höheren Plasma-OxytocinKonzentrationen deskriptiv eine höhere emotionale Bindung und Zustimmung zur Zusammenarbeit in Bezug auf die Therapeutin bzw. die Therapie. Ähnliche Zusammenhänge fanden sich zudem mit den allgemeinen Wirkfaktoren Problemaktualisierung, Ressourcenaktivierung, motivationale Klärung und Problembewältigung [Grawe, 2004]. Diese Ergebnisse liefern erste Hinweise darauf, wie endogene Hormonspiegel mit dem Psychotherapieprozess interagieren können.

Zusammenfassend ergeben sich aus den Beiträgen dieses Themenhefts einige wichtige klinische und wissenschaftliche Implikationen. Aus einer therapeutischen Perspektive betrachtet legitimieren die Artikel zunächst vor allem den Einsatz kognitiv-verhaltenstherapeutischer Interventionen in Bereichen, welche oftmals nicht als eigentliche Kerngebiete der KVT gelten (z.B. Prävention, somatomedizinische Erkrankungen). Die Arbeiten verweisen zudem auf das hohe Potenzial der klinisch-biologischen Forschung, diePlausibilität von mit Patient*innen erarbeiteten Störungsmodellen zu erhöhen sowie auf die Möglichkeit, nachvollziehbarere Behandlungsrationale entwickeln zu können (z.B. in Bezug auf den Einsatz spe- zifischer Techniken wie Stressbewältigung). Durch die Hinweise auf die biologischen Effekte der KVT scheint es schließlich denkbar, dass die Motivation und Adhärenz auf Patient*innenseite gesteigert werden kann (z.B. wenn auf die positiven Effekte der Behandlung in Bezug auf somatomedizinische Komorbiditäten oder Folgeerkrankungen hingewiesen wird). Aus Sicht der Forschung illustrieren die dargestellten Befunde die der KVT zugrundeliegenden Wirkmechanismen und bestätigen in einer "proof-of-concept"-Manier das Wechselspiel zwischen biologischen und psychologischen Faktoren bei der Entstehung von psychischen Störungen und ihren Begleiterscheinungen. Sie zeigen weiter den möglichen Beitrag biologischer Marker zu einer differenzierteren Evaluierung von Psychotherapie über Selbst- und Fremdbeurteilungsmaße hinaus auf. Im Sinne der "precision psychiatry" [Fernandes et al., 2017] liefern sie schließlich Impulse für eine präzisere Differentialdiagnostik und eine Anpassung von Behandlungsplänen an die Bedürfnisse des*r individuellen Patient*in.

\section{Danksagung}

Die Editorinnen bedanken sich herzlich bei allen Gutachter*innen für die konstruktiven Rückmeldungen zu den eingereichten Manuskripten.

\section{Literatur}

Carroll BJ. Use of the dexamethasone suppression test in depression. J Clin Psychiatry. 1982 Nov;43(11 Pt 2):44-50.

Dantzer R, O'Connor JC, Freund GG, Johnson RW, Kelley KW. From inflammation to sickness and depression: when the immune system subjugates the brain. Nat Rev Neurosci. 2008 Jan;9(1):46-56.

Dwyer DB, Falkai P, Koutsouleris N. Machine learning approaches for clinical psychology and psychiatry. Annu Rev Clin Psychol. 2018 May;14(1):91-118.
Eley TC. The future of therapygenetics: where will studies predicting psychological treatment response from genomic markers lead? Depress Anxiety. 2014 Aug;31(8):617-20.

Engel GL. The need for a new medical model: a challenge for biomedicine. Science. 1977 Apr; 196(4286):129-36.

Fernandes BS, Williams LM, Steiner J, Leboyer M, Carvalho AF, Berk M. The new field of "precision psychiatry.” BMC Med. 2017 Apr;15(1): 80.
Grawe K. Neuropsychotherapie. Göttingen: Hogrefe; 2004

Insel T, Cuthbert B, Garvey M, Heinssen R, Pine DS, Quinn K, et al. Research domain criteria (RDoC): toward a new classification framework for research on mental disorders. Am J Psychiatry. 2010 Jul;167(7):748-51.

Lester BM, Tronick E, Nestler E, Abel T, Kosofsky B, Kuzawa CW, et al. Behavioral epigenetics. Ann N Y Acad Sci. 2011 May;1226(1):14-33.

Sies H, Berndt C, Jones DP. Oxidative stress. Annu Rev Biochem. 2017 Jun;86(1):715-48. 